Check for updates

Cite this: RSC Adv., 2019, 9, 8230

Received 12th January 2019

Accepted 24th February 2019

DOI: 10.1039/c9ra00290a

rsc.li/rsc-advances

\section{A novel method for the preparation of solvent-free, microwave-assisted and nitrogen-doped carbon dots as fluorescent probes for chromium(vı) detection and bioimaging $\dagger$}

\author{
Meng Cao, ${ }^{\text {abc }}$ Yong Li, ${ }^{\text {abc }}$ Yunze Zhao, ${ }^{\text {abc }}$ Chongyang Shen, (D) abc Hongyan Zhang (DD d \\ and Yuanfang Huang (D) *abc
}

Chromium(VI) [Cr(vi)] has been shown to be toxic to organisms due to its mutagenicity and carcinogenicity. Therefore, the exploitation of probes with low toxicity and high sensitivity for $\mathrm{Cr}(\mathrm{vl})$ detection is needed. In this study, a one-step, solvent-free, and microwave-assisted method was developed for the preparation of nitrogen-doped carbon dots ( $\mathrm{N}$-CDs). The reaction could be finished in just three minutes, and the yield of the dots could reach 58.5\%; the as-prepared N-CDs exhibited excellent water solubility, emitted bright cyan fluorescence with a high quantum yield of $38.88 \%$, and possessed excitation- and concentrationdependent characteristics. The $\mathrm{N}-\mathrm{CDs}$ could be effectively applied to $\mathrm{Cr}(\mathrm{VI})$ detection with a linear range of 1-100 $\mu \mathrm{M}$, and the detection limit could be as low as $0.12 \mu \mathrm{M}$. The quenching mechanism was responsible for the inner filter effect, and the quenched fluorescence could be recovered with a linear range of 5-100 $\mu \mathrm{M}$ by the addition of ascorbic acid. We showed that the fluorescent probes could even be employed for the detection of $\mathrm{Cr}(\mathrm{VI})$ in river water and for bio-imaging because of their nearly zero cytotoxicity; this showed the potential application of these probes in ion detection and cellular bioimaging. Herein, we have provided an effective strategy to rapidly obtain high-quality $\mathrm{N}$-CDs using a solid-phase microwave method, and the as-prepared N-CDs exhibit various potential applications in environmental and biological fields.

\section{Introduction}

Although the rapid development of industry has significantly contributed to economic growth for the past several decades, it has led to increased levels of environmental pollution. For example, heavy metals are discharged into the environment mainly from industries; once released, the heavy metals can be accumulated in the ecosystems and food chains at extremely high levels because they are hard to be biodegraded and readily form complexes with biological ligands. ${ }^{1,2}$ Chromium $(\mathrm{Cr})$ is one of the most toxic heavy metal ions, and its common valence states are $\mathrm{Cr}(\mathrm{III})$ and $\mathrm{Cr}(\mathrm{VI})$. $\mathrm{Cr}(\mathrm{III})$ has been broadly employed as a dopant in lead titanate nanopowders for multi-frequency electron paramagnetic resonance studies ${ }^{3-5}$ whereas $\mathrm{Cr}(\mathrm{vI})$ is

${ }^{a}$ College of Resources and Environment Sciences, China Agricultural University, Beijing 100193, China. E-mail: yfhuang@cau.edu.cn

${ }^{b}$ Key Laboratory of Agricultural Land Quality, Ministry of Natural Resources, Beijing 100193, China

'Key Laboratory of Arable Land Conservation (North China), Ministry of Agriculture and Rural Affairs, Beijing 100193, China

${ }^{d}$ College of Science, China Agricultural University, Beijing 100193, China

$\dagger$ Electronic supplementary information (ESI) available. See DOI: $10.1039 / \mathrm{c} 9 \mathrm{ra00290a}$ more toxic as compared to $\mathrm{Cr}(\mathrm{III})$ due to its higher solubility in water. Due to its solubility, $\mathrm{Cr}(\mathrm{vI})$ readily enters into the bodies of living organisms and induces mutagenic and carcinogenic risks. ${ }^{6-8}$ As stipulated by the United States Environmental Protection Agency (EPA), the permissible concentration of $\mathrm{Cr}(\mathrm{vI})$ in drinking water has to be under $0.1 \mathrm{mg} \mathrm{L}^{-1}$ (about $1.9 \mu \mathrm{M}$ ). ${ }^{9}$ Therefore, it is of significant importance to develop methods to detect $\mathrm{Cr}(\mathrm{vI})$ at low concentrations with high sensitivity, specific selectivity and quick response.

To date, various analytical techniques, such as spectrophotometry, ${ }^{10,11}$ chromatography, ${ }^{12}$ and electrochemical analysis ${ }^{13,14}$ have been developed for the detection of $\operatorname{Cr}(\mathrm{vI})$. However, in most of these techniques, large instruments are required, and the operation procedures are complex; this greatly limits their applications. Fortunately, carbon dots (CDs), first reported in $2004,{ }^{15}$ have been widely used in supercapacitor applications or as a fluorescent probe due to their good biocompatibility, special optical properties and low cost. ${ }^{16-21}$ Several approaches have been developed for the detection of heavy metal ions based on CDs. ${ }^{22-28}$ For example, Liu et al. ${ }^{23}$ prepared CDs through the hydrothermal treatment of grass to detect cupric ion, and the detection concentration limit could be as low as $1 \mathrm{nM}$. Dang et al. ${ }^{25}$ synthesized highly fluorescent $\mathrm{N}$ 
and S co-doped CDs using high-temperature pyrolysis; these CDs were very effective for the detection of silver ions. Other CDs were obtained by Yang et al. ${ }^{29}$ through a microwaveassisted method using ammonium citrate and a cysteamine hydrochloride solution as raw materials for $\mathrm{Cr}(\mathrm{vr})$ sensing. However, it is still a challenge to develop efficient one-step routes for the large-scale production of CDs using inexpensive materials via green ways; in this regard, microwave-assisted pyrolysis has been applied for the preparation of heteroatomdoped CDs; ${ }^{30-32}$ however, in this method, usually, solvent or liquid reagents are employed to dissolve precursors that are incompatible towards realizing the green and sustainable development. Maleic anhydride (MA), a polar reagent with a low melting point of $52.8{ }^{\circ} \mathrm{C}$, can absorb microwave radiations and easily melt. $^{33,34}$ To the best of our knowledge, MA has seldom been employed as a precursor to produce CDs, and the solidstate synthesis of $\mathrm{N}$-doped CDs without a solvent has been rarely reported.

In this study, we developed a novel method to prepare multifunctional $\mathrm{N}$-doped CDs ( $\mathrm{N}$-CDs) just by MA and urea solids via a one-step microwave treatment (Fig. 1). Then, the fluorescence quenching of these CDs by heavy metal ions was tested. The N-CDs showed sensitive and efficient quenching response to $\mathrm{Cr}(\mathrm{vI})$ at a relatively low concentration level $(0.12$ $\mu \mathrm{M})$, which was much lower than that mentioned in the US EPA stipulation. The emission of the quenched N-CDs could be recovered by adding ascorbic acid (AA) as a reductant (Fig. 1). The produced N-CDs can even be applied for the detection of $\mathrm{Cr}(\mathrm{vI})$ in natural water samples, such as those obtained from river, and can be taken as admirable bio-imaging agents for monitoring $\mathrm{Cr}(\mathrm{vI})$ in plants. We have provided a greener and simpler approach as compared to those reported in the literature using a solid-state synthesis route under microwave irradiation.

\section{Experimental}

\subsection{Materials}

MA, urea, $\mathrm{K}_{2} \mathrm{Cr}_{2} \mathrm{O}_{7}$, ascorbic acid, $\mathrm{NaOH}$ and $\mathrm{HCl}$ were purchased from Aladdin Chemical Co., Ltd (Shanghai, China).
Other chemicals, including $\mathrm{NaCl}, \mathrm{KCl}, \mathrm{AgNO}_{3}, \mathrm{CuCl}_{2} \cdot 2 \mathrm{H}_{2} \mathrm{O}$, $\mathrm{CaCl}_{2} \cdot 2 \mathrm{H}_{2} \mathrm{O}, \mathrm{FeCl}_{2}, \mathrm{MnSO}_{4} \cdot \mathrm{H}_{2} \mathrm{O}, \mathrm{BaCl}_{2}, \mathrm{NiCl}_{2} \cdot 6 \mathrm{H}_{2} \mathrm{O}, \mathrm{MgCl}_{2}$, $\mathrm{Zn}\left(\mathrm{NO}_{3}\right)_{2}, \mathrm{FeCl}_{3} \cdot 6 \mathrm{H}_{2} \mathrm{O}, \mathrm{Al}\left(\mathrm{NO}_{3}\right)_{3} \cdot 9 \mathrm{H}_{2} \mathrm{O}$ and $\mathrm{CrCl}_{3} \cdot 6 \mathrm{H}_{2} \mathrm{O}$, were obtained from Sinopharm Chemical Reagent Co., Ltd (Shanghai, China). Mung bean seeds were purchased from retail outlets. All the chemical agents were of analytical grade and used as received without further purification. Deionized water was used for preparing the solutions.

\subsection{Synthesis of N-doped carbon dots (N-CDs)}

The N-CDs were synthesized in one step using a green, microwave-assisted, and solvent-free method. Specifically, a solid mixture of $1.18 \mathrm{~g}$ MA and $1.08 \mathrm{~g}$ urea was ground into a fine powder in an agate mortar. The mixture was transferred to a beaker and allowed to react for three minutes in a modified domestic $700 \mathrm{~W}$ microwave oven. The oven was equipped with a sensor to determine the change in pressure and temperature per five seconds. During the reaction, the mixture changed from white to brown and finally became dark brown. The mixture was then cooled down to room temperature, and $50 \mathrm{~mL}$ deionized water was added to the solid mixture. To remove large particles, the obtained solution was centrifuged at $10000 \mathrm{rpm}$ for ten minutes and filtered using a $0.22 \mu \mathrm{m}$ membrane. The resulting brownish yellow solution was dialyzed using a dialysis tube $(\mathrm{MWCO}=1000 \mathrm{Da})$ for $24 \mathrm{~h}$ and freeze-dried to obtain browncolored solid N-CDs.

\subsection{Characterization}

The size and morphology of the N-CDs were examined by the $\mathrm{H}$ 600 transmission electron microscope (TEM, Hitachi, Ltd). The surface structure of the N-CDs was determined by Fourier transform infrared (FTIR) spectroscopy and X-ray photoelectron spectroscopy (XPS); the FTIR spectra were obtained using the Bruker VERTEX 70 FTIR spectrometer (Bruker Daltonics Inc.), and the XPS spectra were acquired using the Kratos AXIS ULTRA DLD X-ray photoelectron spectrometer (Shimadzu Corp.); the optical properties of the N-CDs were investigated by ultraviolet visible (UV-Vis) absorption spectroscopy, fluorescence spectrogram and fluorescence microscopy. The UV-Vis spectra were

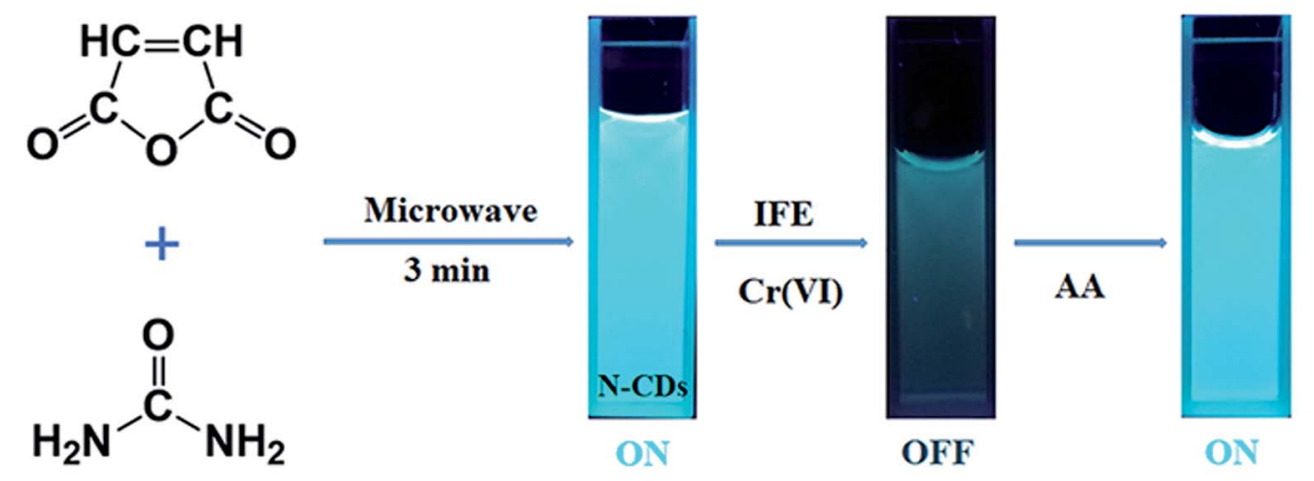

Fig. 1 Schematic of the synthesis of the $\mathrm{N}$-doped $\mathrm{CDs}(\mathrm{N}-\mathrm{CDs})$ and the on-off-on detection of chromium(VI) [Cr(VI)] and ascorbic acid (AA) based on the inner filter effect (IFE). The fluorescence images were obtained under $365 \mathrm{~nm}$ UV light. ON: fluorescent N-CDs and OFF: quenched $\mathrm{N}-\mathrm{CDs}$. 
obtained using Evolution 300 (Thermo Electron Corp.). All the three-dimensional (3D) spectrograms, emission spectra, absolute quantum yields (QYs) and fluorescence lifetimes were obtained using the Fluoromax-3 spectrofluorometer (HORIBA JY Inc). The absolute QY was measured using an integral sphere. The fluorescence images were obtained using the OLYMPUS BX51 fluorescence microscope (Olympus Corp.).

\subsection{Detection of heavy metal ions}

Herein, $1 \mathrm{~g}$ of the as-prepared $\mathrm{N}-\mathrm{CDs}$ was dissolved in $1 \mathrm{~L}$ deionized water to achieve a concentration of $1.0 \mathrm{mg} \mathrm{mL}$. Then, the aqueous solutions involving fifteen kinds of metal ions $\left[\mathrm{Cr}(\mathrm{VI}), \mathrm{Na}^{+}, \mathrm{K}^{+}, \mathrm{Ag}^{+}, \mathrm{Cu}^{2+}, \mathrm{Ca}^{2+}, \mathrm{Fe}^{2+}, \mathrm{Mn}^{2+}, \mathrm{Ba}^{2+}, \mathrm{Ni}^{2+}, \mathrm{Mg}^{2+}\right.$, $\mathrm{Zn}^{2+}, \mathrm{Fe}^{3+}, \mathrm{Al}^{3+}$, and $\left.\mathrm{Cr}^{3+}\right]$ were prepared at the concentration of $1 \mathrm{mM}$. To test the selectivity of the N-CDs, $0.5 \mathrm{~mL}$ of the abovementioned metal ion solution was blended with $1.0 \mathrm{~mL} \mathrm{N-}$ $\mathrm{CD}$ aqueous solution, and the mixture was diluted to $10.0 \mathrm{~mL}$ with deionized water. The fluorescence emission spectra were obtained after one-minute incubation at a wavelength of $360 \mathrm{~nm}$. The sensitivity of the N-CDs to $\mathrm{Cr}(\mathrm{vI})$ was tested by mixing $1.0 \mathrm{~mL}$-CD solution and a proper volume of the $\mathrm{Cr}(\mathrm{vI})$ solution. The blended solution was attenuated to $10 \mathrm{~mL}$, and its fluorescence spectrum was obtained after one minute at the excitation of $360 \mathrm{~nm}$. All the experiments were carried out at $27^{\circ} \mathrm{C}$.

\subsection{Detection of $\mathrm{Cr}(\mathrm{vI})$ in a real sample}

To evaluate the N-CD-based sensor for the $\mathrm{Cr}(\mathrm{vI})$ detection in practical application, real water sample measurement was performed using the river water (E $\left.107^{\circ} 38^{\prime} 3^{\prime \prime}, \mathrm{N} 24^{\circ} 50^{\prime} 43^{\prime \prime}\right)$ of Guangxi Province, China. The river water was centrifuged at $10000 \mathrm{rpm}$ for ten minutes followed by filtration through a 0.22 $\mu \mathrm{m}$ membrane. Then, the sample was added to the N-CD and $\mathrm{Cr}(\mathrm{vI})$ aqueous solution containing different concentrations of $\mathrm{Cr}(\mathrm{vI})$, and the fluorescence intensity was determined.

\subsection{Multicolor bioimaging}

The mung bean seeds were used to grow sprouts for the multicolor bioimaging. Herein, three groups of seeds, labeled as 1 st, 2nd and 3rd, were used, and each group included 100 seeds. These mung bean seeds were added to deionized water and placed in a plastic plate with holes in the bottom. The seeds were covered with a white cloth and kept in the dark at $27^{\circ} \mathrm{C}$ for 48 hours. Deionized water was employed during the breeding process to remove impurities. After 48 hours of incubation, deionized water was replaced with the N-CD solution $(0.1 \mathrm{~g}$ $\left.\mathrm{mL}^{-1}\right)$. After 4 hours, the $\operatorname{Cr}(\mathrm{vI})$ solution $(50 \mu \mathrm{M})$ was added to the 2nd and 3rd samples. After another four hours, the AA solution $(100 \mu \mathrm{M})$ was poured into the 3rd sample. The mung bean sprouts were reaped after a total of 60 hours' cultivation, followed by washing with deionized water to remove the residual agents for fluorescence imaging.

\section{Results and discussion}

\subsection{Characterization of the N-CDs}

In this study, new fluorescent N-CDs with high yields were synthesized using MA and urea as precursors via a solvent-free, solid-state and microwave-assisted pyrolysis route. As illustrated in Fig. 1, the reaction completed in just three minutes, and the acquired N-CDs had a high yield of $58.5 \%$. Interestingly, the pressure of this reaction remained constant throughout the experiments, whereas the temperature changed. As shown in Fig. 2a, the reaction temperature slowly increased from room temperature to $80{ }^{\circ} \mathrm{C}$ in the first 135 seconds. After this, the temperature sharply increased from $80{ }^{\circ} \mathrm{C}$ to $228{ }^{\circ} \mathrm{C}$ in just 30 seconds and remained almost steady in the final 15 seconds; this indicated the end of the reaction. Fig. $2 b$ shows the TEM image of the morphology and structure of the N-CDs; the asprepared N-CDs were nearly orbicular with a very narrow size distribution $(3.0-6.8 \mathrm{~nm})$ and an average diameter of $5.3 \mathrm{~nm}$. The high-resolution TEM image of the N-CDs, as shown in Fig. $2 \mathrm{~b}$, indicates that the prepared N-CDs exhibit the lattice fringe of $0.21 \mathrm{~nm}$, which is consistent with the (100) facet of graphitic carbon..$^{35}$ The XRD pattern (Fig. S1 $\dagger$ ) showed that the $\mathrm{N}$-CDs exhibited an amorphous graphite structure. This is likely because the existence of oxygen- and nitrogen-containing functional groups causes a significant disorder in N-CDs.

FTIR spectroscopy and XPS were used to determine the surface structure of the N-CDs. As shown in Fig. S2a, $\uparrow$ the broad peak range of $2800-3480 \mathrm{~cm}^{-1}$ in the FTIR spectrum of the $\mathrm{N}$ -
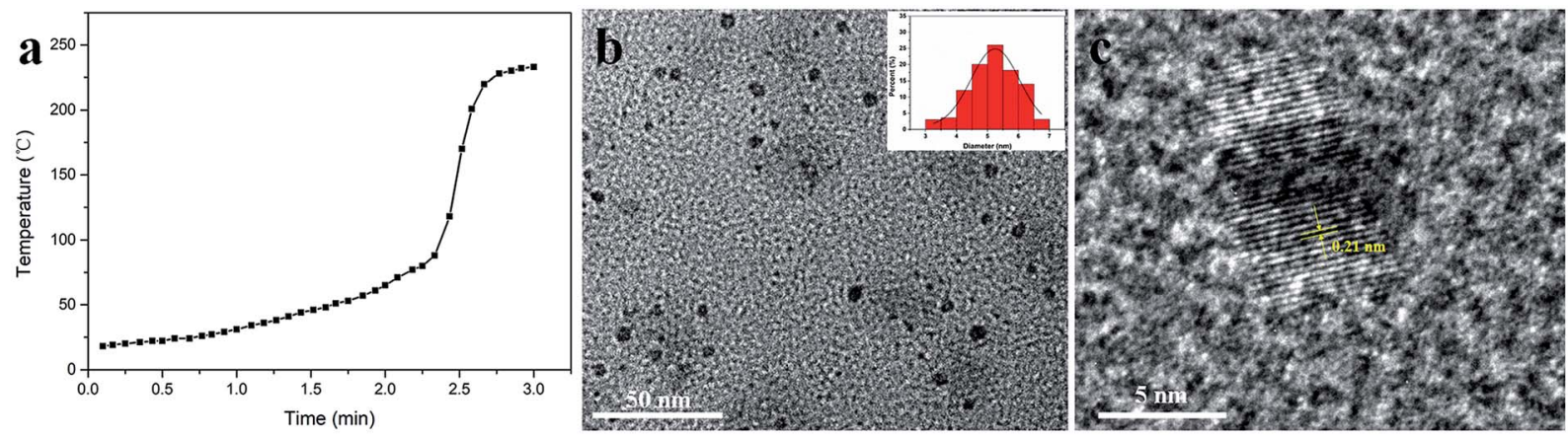

Fig. 2 (a) Relationship between the temperature and the time. Data were obtained per five seconds. (b) A TEM image of the N-CDs. Inset: size distribution. (c) High-resolution TEM image of the N-CDs, and lattice fringes of a single N-CD. 
CDs was due to the $\mathrm{N}-\mathrm{H}$ and $-\mathrm{OH}$ stretching vibration. ${ }^{36}$ In addition, the two significant peaks at $1715 \mathrm{~cm}^{-1}$ and $1635 \mathrm{~cm}^{-1}$ indicated the presence of $\mathrm{C}=\mathrm{O}$ and $\mathrm{C}=\mathrm{C}$ vibrations, respectively. ${ }^{37}$ The other two absorption bands at $1440 \mathrm{~cm}^{-1}$ and $1256 \mathrm{~cm}^{-1}$ were assigned to the $\mathrm{C}-\mathrm{N}$ and $\mathrm{C}-\mathrm{O}$ vibrations, respectively. ${ }^{38}$ The elemental composition of the N-CDs was further characterized by XPS. Fig. $\mathrm{S} 2 \mathrm{~b} \dagger$ shows three dominant peaks at $285.8 \mathrm{eV}\left(\mathrm{C}_{1 \mathrm{~s}}\right), 399.8 \mathrm{eV}\left(\mathrm{N}_{1 \mathrm{~s}}\right)$, and $534.2 \mathrm{eV}\left(\mathrm{O}_{1 \mathrm{~s}}\right)$, indicating the presence of $\mathrm{C}, \mathrm{N}$ and $\mathrm{O}$, respectively. The atomic percentage contents were $38.19 \%$ for $\mathrm{C}, 18.68 \%$ for $\mathrm{N}$, and $43.13 \%$ for $\mathrm{O}$ (see inset in Fig. $\mathrm{S} 2 \mathrm{~b} \dagger$ ). The results indicated the formation of highly N-doped, O-rich N-CDs with MA and urea as raw materials. The high-resolution $\mathrm{C}_{1 \mathrm{~s}}, \mathrm{~N}_{1 \mathrm{~s}}$ and $\mathrm{O}_{1 \mathrm{~s}}$ spectra are presented in Fig. 3a-c. The $\mathrm{C}_{1 \mathrm{~s}}$ spectrum could be deconvoluted into four peaks located at 284.1, 284.8, 285.7 and $288.4 \mathrm{eV}$, corresponding to the $\mathrm{C}-\mathrm{C} / \mathrm{C}=\mathrm{C}, \mathrm{C}-\mathrm{N}, \mathrm{C}-\mathrm{O}$ and $\mathrm{C}=\mathrm{O}$ groups. ${ }^{39}$ The $\mathrm{N}_{1 \mathrm{~s}}$ peak exhibited three different states of $\mathrm{N}$ : pyridinic $\mathrm{N}$ at $399.4 \mathrm{eV}$, pyrrolic $\mathrm{N}$ at $400.2 \mathrm{eV}$ and graphitic $\mathrm{N}$ at $401.1 \mathrm{eV} \mathbf{;}^{\mathbf{4 0}}$ in the $\mathrm{O}_{1 \mathrm{~s}}$ spectrum, the two optimized peaks at 531.6 and $531.8 \mathrm{eV}$ corresponded to the $\mathrm{O}=\mathrm{C}$ and $-\mathrm{OH} / \mathrm{C}-\mathrm{O}-\mathrm{C}$ groups; ${ }^{41}$ the FTIR and XPS results demonstrated that the as-prepared N-CDs contained a significant number of oxygen- and nitrogencontaining groups; therefore, the probable molecular structure of the N-CDs could be proposed, as illustrated in Fig. 3d.
After this, the optical properties of the N-CDs were investigated by UV-Vis absorption spectroscopy and 3D fluorescence spectrograms. Fig. 4a shows that there were two absorbance bands appearing at 260 and $340 \mathrm{~nm}$, which could be attributed to the $\pi-\pi^{*}$ transition of the $\mathrm{C}=\mathrm{C} \mathrm{sp}{ }^{2}$ domains in the carbon core and the $n-\pi^{*}$ transition of the $\mathrm{C}=\mathrm{O} / \mathrm{C}-\mathrm{N}$ moieties on the surface, respectively. ${ }^{42}$ The N-CD aqueous solution was faint yellow in the daylight and became bright cyan under $365 \mathrm{~nm}$ UV light (inset in Fig. 4a). The 3D fluorescence spectra of the abovementioned N-CD solution showed a strong emission range of 355-660 $\mathrm{nm}$ at the broad excitation wavelength of 250$500 \mathrm{~nm}$ (Fig. 4b) due to the inhomogeneous size distribution and multiple groups around the nanodots. The maximum emission peak was located at $484 \mathrm{~nm}$ under excitation at $360 \mathrm{~nm}$, and the emission peak regularly moved to longer wavelength with an increase in the excitation wavelength (Fig. S3a $\dagger$ ); furthermore, the 3D fluorescence spectra for the concentrations 0.01 and $1 \mathrm{mg} \mathrm{mL} \mathrm{m}^{-1}$ of the $\mathrm{N}$-CDs are presented in Fig. S3b and $\mathrm{c} \uparrow$ to show the influence of the concentration of the N-CDs. When the concentration was below $0.1 \mathrm{mg} \mathrm{mL}^{-1}$, the emission almost remained unchanged. In contrast, the maximum excitation and emission wavelength red-shifted at $1 \mathrm{mg} \mathrm{mL}{ }^{-1}$; this was attributed to the presence of multiple fluorescent centers in the N-CDs and the enhancement of
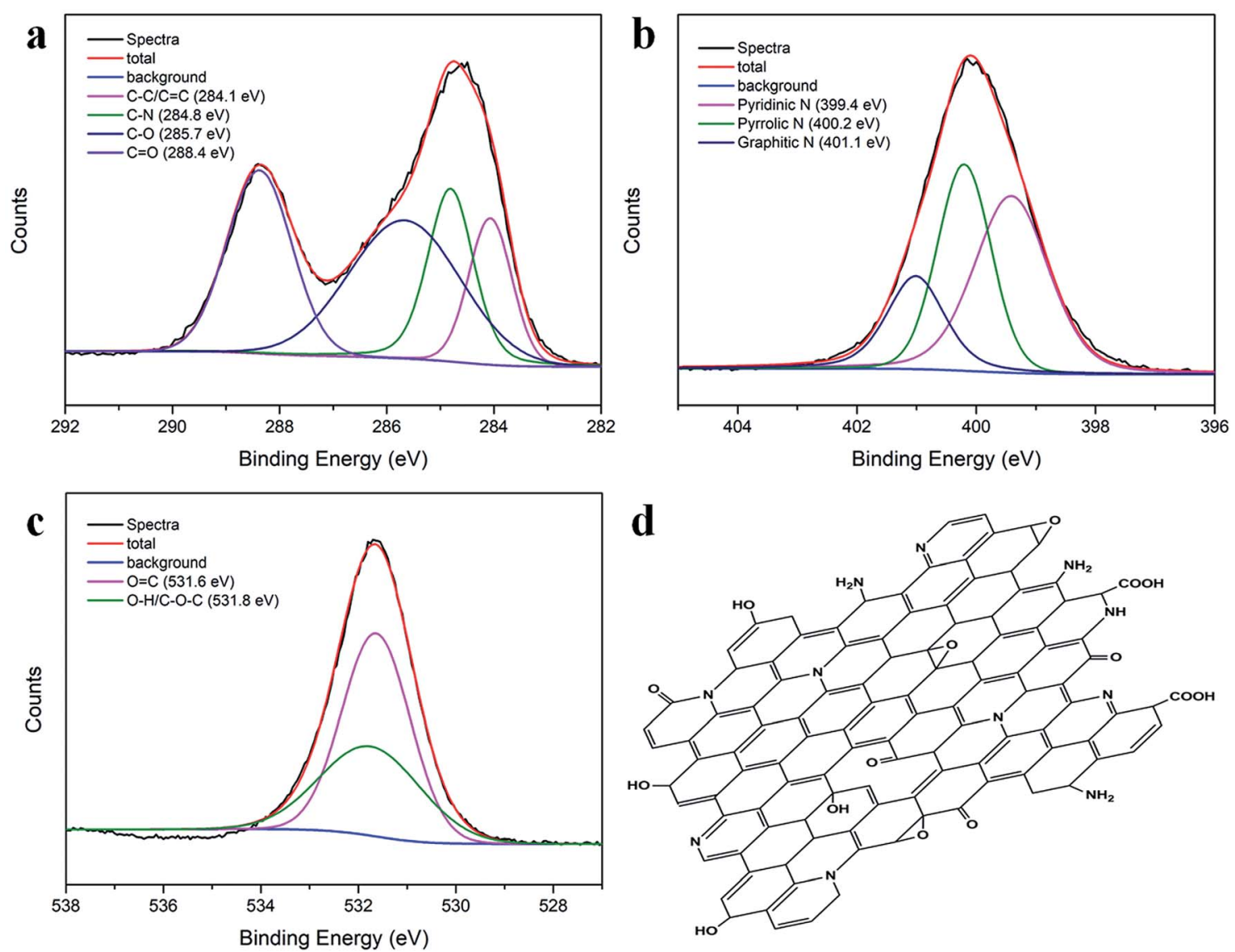

Fig. 3 XPS spectra of the (a) $\mathrm{C}_{1 \mathrm{~s}}$ (b) $\mathrm{N}_{1 \mathrm{~s}}$ and (c) $\mathrm{O}_{1 \mathrm{~s}}$ of $\mathrm{N}-\mathrm{CD}$. Spectra represented the measured spectra, the total was the fitted curve, background meant the baseline of the measured spectra, and other spectra were obtained by deconvoluting the corresponding spectra. (d) The probable molecular structure of the N-CDs. 

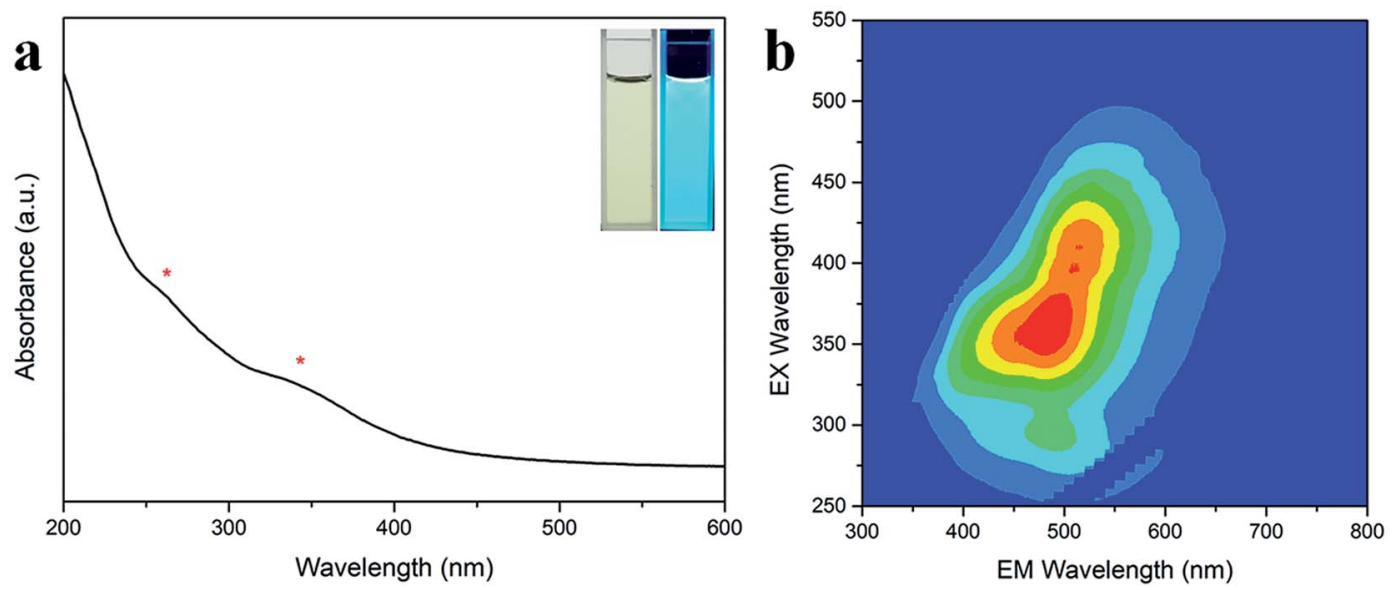

Fig. 4 (a) UV-Vis absorption spectrum of the N-CDs. *Two absorbance bands at 260 and $340 \mathrm{~nm}$. Inset: images of the N-CD solution in daylight (left) and under $365 \mathrm{~nm}$ UV light (right). (b) The 3D fluorescence spectrogram of the N-CDs. Fluorescence intensity increased with color change from blue to red.

emission resorption. ${ }^{43-45}$ The absolute QY values for $0.01,0.1$ and $1 \mathrm{mg} \mathrm{mL}^{-1}$ were calculated to be $23.02 \%, 38.88 \%, 10.36 \%$, respectively. Therefore, $0.1 \mathrm{mg} \mathrm{mL}^{-1}$ was the optimal concentration of N-CDs.

The N-CD solution also exhibited excellent stability against UV light, $\mathrm{pH}$ values and ionic strengths. As illustrated in Fig. S4a and $b, \dagger$ the fluorescence intensity could be retained up to $90 \%$ after one hour of UV irradiation, and the N-CDs were relatively stable when the $\mathrm{pH}$ varied between 3.0 and 8.0. In addition, no obvious fluctuation was observed in the fluorescence intensity when the $\mathrm{NaCl}$ concentration was increased from 0 to $1 \mathrm{M}$ (Fig. S4c $\dagger$ ). All these results indicated the competent solventfree synthesis of efficient, highly stable and cyan-fluorescent N-CDs.

\subsection{Selectivity of the N-CDs for $\mathrm{Cr}(\mathrm{vI})$}

To explore the selectivity for $\mathrm{Cr}(\mathrm{vI})$ detection, the fluorescence intensities of the N-CDs with $\mathrm{Cr}(\mathrm{VI})$ and other fourteen metal ions $\mathrm{Na}^{+}, \mathrm{K}^{+}, \mathrm{Ag}^{+}, \mathrm{Cu}^{2+}, \mathrm{Ca}^{2+}, \mathrm{Fe}^{2+}, \mathrm{Mn}^{2+}, \mathrm{Ba}^{2+}, \mathrm{Ni}^{2+}, \mathrm{Mg}^{2+}, \mathrm{Zn}^{2+}$, $\mathrm{Fe}^{3+}, \mathrm{Al}^{3+}$ and $\left.\mathrm{Cr}^{3+}\right)$ were investigated under the maximum excitation of $360 \mathrm{~nm}$. These metal ions were used at same concentrations $(50 \mu \mathrm{M})$. The fluorescence relative change ratio $F_{0}-F$ is illustrated in Fig. 5, and $F_{0}$ and $F$ are defined as the fluorescence intensities of the N-CD solution without and with the presence of metal ions, respectively. The results indicated that obvious fluorescence quenching occurred with the addition of $\mathrm{Cr}(\mathrm{vI})$, whereas insignificant changes were observed for other ions. These results demonstrate that the prepared $\mathrm{N}$-CDs are effective to be used as a fluorescent sensor for the detection of $\mathrm{Cr}(\mathrm{vI})$ with high selectivity.

\subsection{Sensitivity of the N-CDs for $\mathrm{Cr}(\mathrm{vI})$}

The quenching ability of $\mathrm{Cr}(\mathrm{vI})$ on N-CDs was measured by adding the $\mathrm{Cr}(\mathrm{vI})$ aqueous solution at different concentrations. As displayed in Fig. 6a, the fluorescence intensity of the N-CDs distinctly decreased with an increase in the $\mathrm{Cr}(\mathrm{vI})$ concentration from 0 to $600 \mu \mathrm{M}$, and a slight red-shift occurred in the emission peaks. The inset in Fig. 6a shows that a monoexponential relationship exists between the quenching efficiency $\left(F_{0} / F\right)$ and the concentration of $\mathrm{Cr}(\mathrm{vI})$. The exponential equation was fitted to be $F_{0} / F=1.44 \times \exp (C / 243.86)-0.45(C$ is the $\operatorname{Cr}(\mathrm{vi})$ concentration) for the $\mathrm{Cr}(\mathrm{vI})$ concentration at $1-600 \mu \mathrm{M}$ with $R^{2}$ $=0.9998$. In addition, there was a linear relationship that could be fitted by the Stern-Volmer (SV) equation $F_{0} / F=1+K_{\mathrm{SV}} \times C$ (Fig. 6b), where $K_{\mathrm{SV}}$ is the SV quenching constant, and $C$ is the $\mathrm{Cr}(\mathrm{vI})$ concentration. The linear interval was $1-100 \mu \mathrm{M}$ with an $R^{2}$ of 0.9965 , and the limit of detection (LOD) was $0.12 \mu \mathrm{M}$ based on $3 \sigma / s$ ( $\sigma$ is the deviation of the blank signal, and $s$ is the slope of the SV equation).$^{29}$ Herein, the LOD for $\mathrm{Cr}(\mathrm{vI})$ sensing was much lower than the maximum contaminant level limited by the US EPA; ${ }^{9}$ this demonstrated that the proposed approach based on the N-CD fluorescent probes could have potential application in the detection of $\mathrm{Cr}(\mathrm{vI})$ in drinking water.

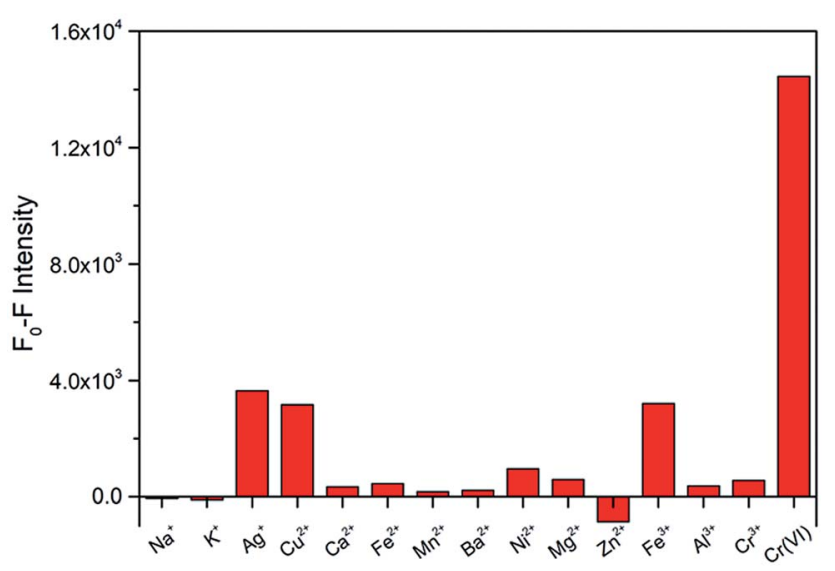

Fig. 5 Fluorescence intensity changes of the N-CDs for various metal ions in water. The concentrations of all the metal ions were $50 \mu \mathrm{M}$. $F_{0}$ and $F$ are the fluorescence intensities of the $\mathrm{N}-\mathrm{CDs}$ without and with the addition of metal ions. 

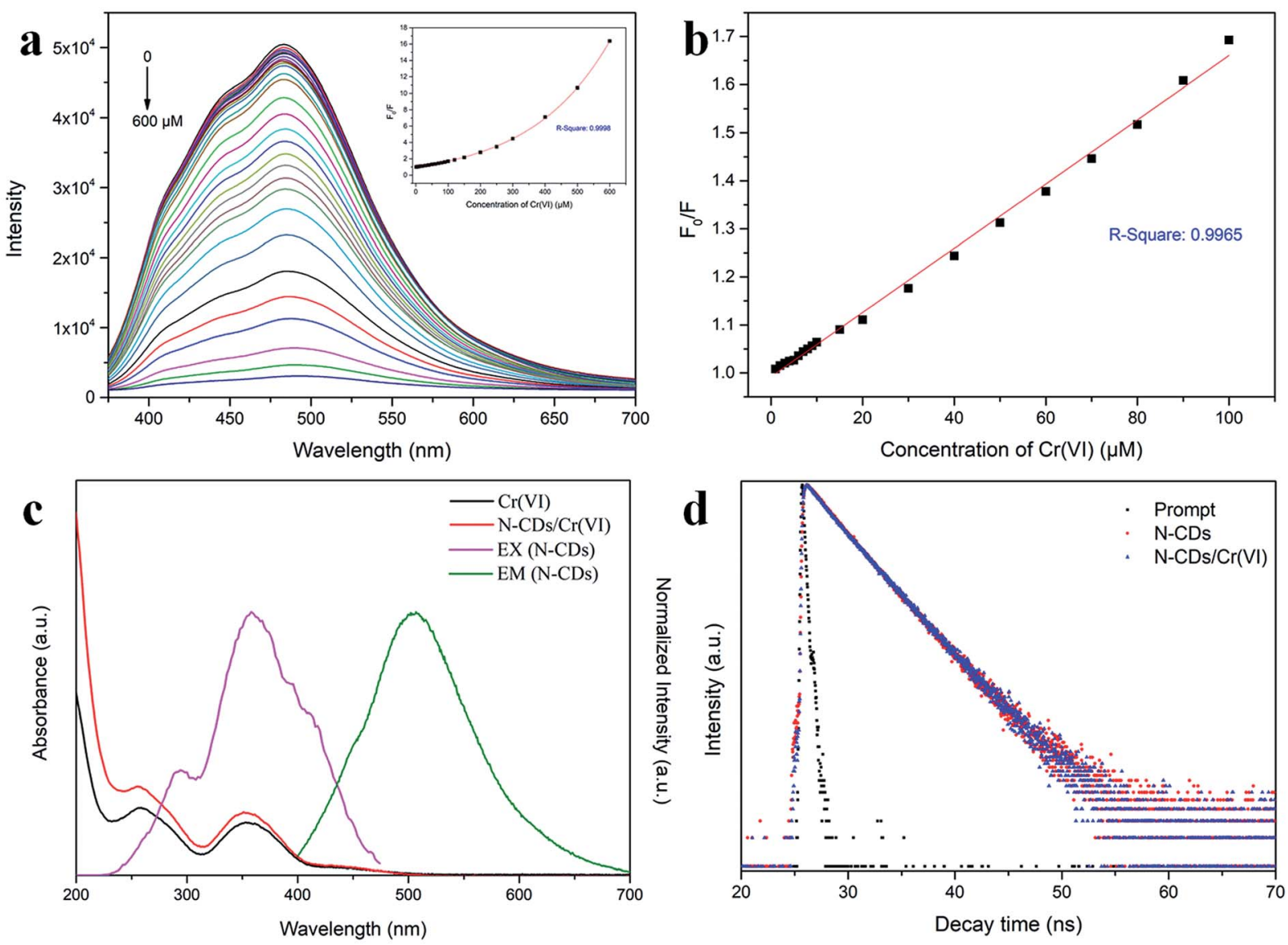

Fig. 6 (a) Fluorescence emission spectra of the $\mathrm{N}-\mathrm{CDs}$ at the $\mathrm{Cr}(\mathrm{VI})$ concentration of $0-600 \mu \mathrm{M}$ at the excitation wavelength of $360 \mathrm{~nm}$. Inset shows the monoexponential variation of the quenching efficiency $\left(F_{0} / F\right)$ with $\mathrm{Cr}(\mathrm{VI})$ concentration. $F_{0}$ and $F$ are the emission intensities of the $\mathrm{N}$ $\mathrm{CDs}$ in the absence and presence of $\mathrm{Cr}(\mathrm{VI})$. (b) The linear variation of $F_{0} / F$ with the $\mathrm{Cr}(\mathrm{VI})$ concentration in the range of 1-100 $\mu \mathrm{M}$. (c) The UV-Vis absorption spectra of $\mathrm{Cr}(\mathrm{VI})$ and $\mathrm{N}-\mathrm{CD} / \mathrm{Cr}(\mathrm{VI})$, the excitation spectrum of $\mathrm{N}-\mathrm{CDs}$ at the emission wavelength of $484 \mathrm{~nm}$, and the emission spectrum of N-CDs at the excitation wavelength of $360 \mathrm{~nm}$. (d) The decay curves of the N-CDs and N-CD/Cr(v). Prompt was blank scanning. The excitation and probed wavelengths were $360 \mathrm{~nm}$ and $484 \mathrm{~nm}$, respectively.

\subsection{The quenching mechanism of N-CDs}

The quenching mechanisms of the probes prepared by the fluorescent CDs generally include intramolecular charge transfer (ICT), ${ }^{46}$ photoinduced electron transfer (PET), ${ }^{47}$ fluorescence resonance energy transfer (FRET) ${ }^{48}$ inner filter effect (IFE) ${ }^{49}$ and so forth. These mechanisms are due to the intermolecular interactions between the fluorescent CDs and target material, causing the variation of radiative relaxation in the excited electrons and accordingly strengthening or weakening of the fluorescence emission.

To explore the fluorescence quenching mechanism by $\mathrm{Cr}(\mathrm{vI})$, the UV-Vis spectra of $\mathrm{Cr}(\mathrm{vI})$ and $\mathrm{N}-\mathrm{CD} / \mathrm{Cr}(\mathrm{vI})$ and the fluorescence excitation and emission spectrograms of the N-CDs were obtained, as shown in Fig. 6c. The absorption spectra of $\mathrm{Cr}(\mathrm{vI})$ and $\mathrm{N}-\mathrm{CD} / \mathrm{Cr}(\mathrm{vI})$ exhibited two same peaks located at $260 \mathrm{~nm}$ and $355 \mathrm{~nm}$, whereas the excitation and emission spectra of the $\mathrm{N}$ CDs were centered at $360 \mathrm{~nm}$ and $484 \mathrm{~nm}$, respectively. This meant that the absorption region of $\mathrm{Cr}(\mathrm{vI})$ had certain overlap with the excitation and emission areas of the N-CDs because some excitation energy of the N-CDs was subdued, and some emission was resorbed by $\mathrm{Cr}(\mathrm{vr})$. These results suggested that the quenching mechanism was based on the IFE theory in our study. To further confirm this mechanism, the lifetimes of the $\mathrm{N}$-CDs and $\mathrm{N}-\mathrm{CD} / \mathrm{Cr}(\mathrm{vI})$ were investigated by time-correlated single-photon counting (TCSPC), and the results are shown in Fig. $6 \mathrm{~d}$. The average lifetimes of the N-CDs in the absence and presence of $\mathrm{Cr}(\mathrm{vI})$ excited at $360 \mathrm{~nm}$ and probed at $484 \mathrm{~nm}$ were calculated to be $3.31 \mathrm{~ns}$ and $3.39 \mathrm{~ns}$, respectively. The microclose data confirmed that the quenching mechanism of $\mathrm{Cr}(\mathrm{vI})$ by our prepared N-CDs was due to the IFE with a static quenching.

\subsection{Fluorescence "off-on" by ascorbic acid (AA)}

$\mathrm{AA}$, known as water-soluble vitamin $\mathrm{C}$, is widely used as an antioxidative agent in managing heavy metal ion pollution. The reduction treatment $\mathrm{Cr}(\mathrm{VI})$ using $\mathrm{AA}$ has received significant attention. Fig. 7a shows the recoverability of the quenched fluorescent $\mathrm{N}-\mathrm{CD} / \mathrm{Cr}(\mathrm{vI})$ upon the gradual addition of AA. For the $\mathrm{N}-\mathrm{CD} / \mathrm{Cr}(\mathrm{VI})$ system, $\mathrm{Cr}(\mathrm{vI})$ could be reduced to $\mathrm{Cr}(\mathrm{III})$ by AA, the IFE was faded away, and the emission of the N-CDs was resumed. ${ }^{50}$ Compared with that of the original N-CD sample, the recovery of the emission of $\mathrm{N}-\mathrm{CD} / \mathrm{Cr}(\mathrm{vI})$ could reach $98.9 \%$ 

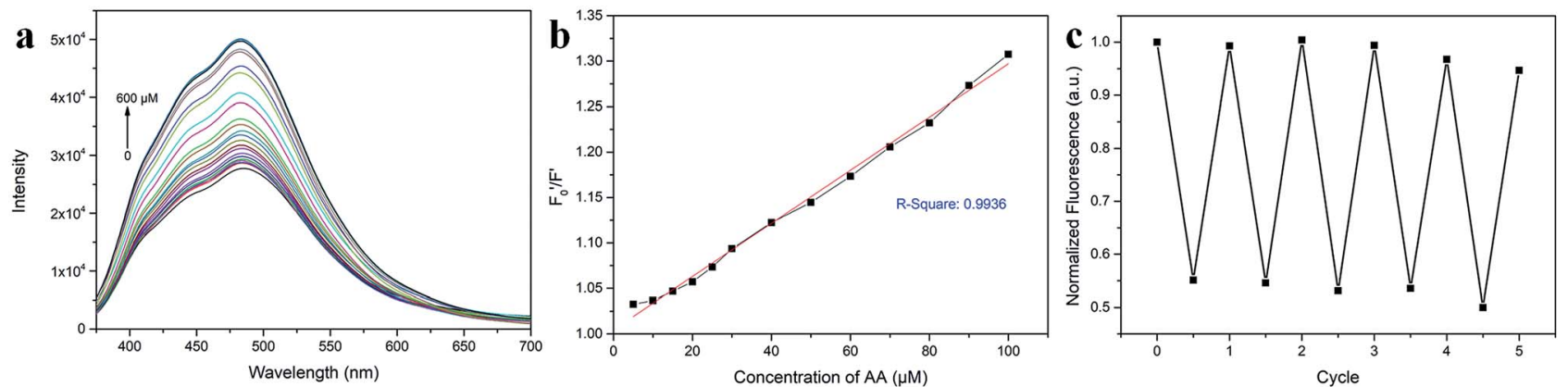

Fig. 7 (a) Fluorescence emission spectra of the $\mathrm{N}-\mathrm{CD} / \mathrm{Cr}(\mathrm{vI})$ with different AA concentrations of $0-600 \mu \mathrm{M}$ at the excitation wavelength of $360 \mathrm{~nm}$. (b) The linear relationship between the recovery efficiency $\left(F_{0}^{\prime} / F^{\prime}\right)$ and AA concentration in the range of 5-100 $\mu \mathrm{M}$. $F_{0}^{\prime}$ and $F^{\prime}$ are the fluorescence intensities of $\mathrm{N}-\mathrm{CD} / \mathrm{Cr}(\mathrm{VI})$ with and without the addition of $\mathrm{AA}$. (c) Fluorescence reproducibility of the $\mathrm{N}-\mathrm{CD}$ solution by the addition of $\mathrm{Cr}(\mathrm{VI})$ and $\mathrm{AA}$. The original fluorescence of $\mathrm{N}-\mathrm{CD}$ solution was normalized to 1.

when the concentration of AA was at $600 \mu \mathrm{M}$. Moreover, the recovery efficiency $\left(F_{0}^{\prime} / F^{\prime}\right)$ could linearly fit the SV equation $\left(R^{2}\right.$ $=0.9946, \mathrm{LOD}=2.72 \mu \mathrm{M}$ ) with the AA concentration increasing from $5 \mu \mathrm{M}$ to $100 \mu \mathrm{M}$, where $F_{0}^{\prime}$ and $F^{\prime}$ are the emission intensity of $\mathrm{N}-\mathrm{CD} / \mathrm{Cr}(\mathrm{vI})$ with and without the existence of AA (Fig. $7 \mathrm{~b}$ ); in addition, we found that the fluorescence intensity of the N-CD solution could remain up to $96 \%$ when the N-CD solution was quenched by $\mathrm{Cr}(\mathrm{vI})$ and recovered by AA for four cycles; this proved the wonderful fluorescence reproducibility of these $\mathrm{N}$ CDs (Fig. 7c). Hence, the N-CD fluorescent sensor with the "on-off-on" property was developed simply by alternately adding $\mathrm{Cr}(\mathrm{VI})$ and $\mathrm{AA}$.

\subsection{Performances of the CDs}

The analytical performance of the present CDs was compared with that of some reported fluorescent CDs for $\mathrm{Cr}(\mathrm{vI})$ detection. Table 1 lists the type of fluorescent probe, synthesized methods, solvent used in the reaction, linear range of $\mathrm{Cr}(\mathrm{vI})$ detection, LODs and QYs. ${ }^{29,36,51-55}$ Microwave-assisted pyrolysis is a facile and highly efficient method than other synthetic methods due to it time-saving, energy-conserving, cost-effective, and convenient operation. ${ }^{32}$ However, many methods have been conducted under hydrothermal conditions, ${ }^{32,51-54}$ which need longer reaction time and consume more energy than the microwave method. Although some studies have used the microwave-assisted methods, ${ }^{29,55}$ solvent or liquid reagents have been employed in them to dissolve the precursors (Table 1), which are incompatible towards achieving the green and sustainable development. Furthermore, the linear range of the $\mathrm{Cr}(\mathrm{vI})$ detection, LODs and QYs for the N-CD fluorescent probe in this study were better.

Therefore, using microwave irradiation, we have provided a novel, solvent-free and solid-state method that is greener and simpler than the existing approaches. Our experimental results indicated that this proposed approach is adequate for $\mathrm{Cr}(\mathrm{vI})$ detection.

For testifying the practical performance of this N-CD-based probe, the samples of river water obtained from Guangxi Province were used for $\mathrm{Cr}(\mathrm{vI})$ detection. The river water was boiled at first and then filtered using a $0.22 \mu \mathrm{m}$ membrane. The detection results are listed in Table 2 . These data exhibited that the spiked recoveries were $98.2-102.6 \%$, and the relative standard deviation (RSD) was below $3 \%$. Consequently, the as-prepared N-CD fluorescent probe was sensitive to $\mathrm{Cr}(\mathrm{vI})$; this suggested the potential possibility of applying the N-CD probes in real water detection.

\subsection{Fluorescence bioimaging}

CDs are famous for their low toxicity, excellent biocompatibility, and good water-solubility, and they have been applied in bioimaging. ${ }^{17,56}$ Herein, mung bean sprouts were employed as the fluorescence matrix. The sprouts could still grow up healthily after the addition of the N-CD solution; this demonstrated the low cytotoxicity of the as-prepared N-CDs. Fig. 8a1-a5 show the

Table 1 Comparison of the performances of the CDs prepared in this study and those reported in the literature for the Cr(vI) detection

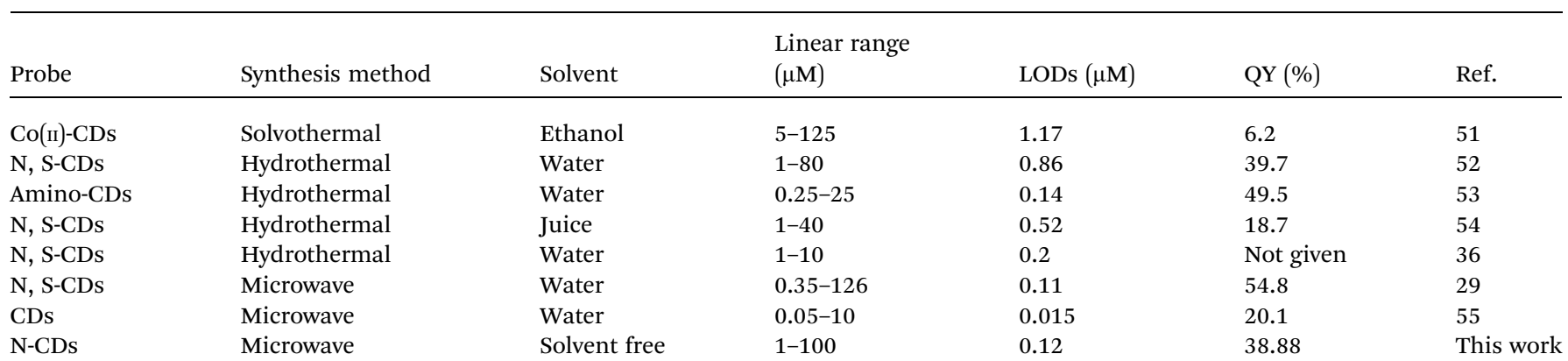


Table 2 The results of detection of $\mathrm{Cr}(\mathrm{VI})$ in river water $(n=3)^{a}$

\begin{tabular}{llclc}
\hline Sample & Found $(\mu \mathrm{M})$ & Added $(\mu \mathrm{M})$ & Total found $(\mu \mathrm{M})$ & Recovery $(\%)$ \\
\hline 1 & Not detected & 0 & Not detected & - \\
2 & Not detected & 5 & 5.13 & 102.6 \\
3 & Not detected & 50 & 49.68 & 99.4 \\
4 & Not detected & 100 & 98.18 & 98.2
\end{tabular}

${ }^{a} \mathrm{RSD}$ : relative standard deviation.

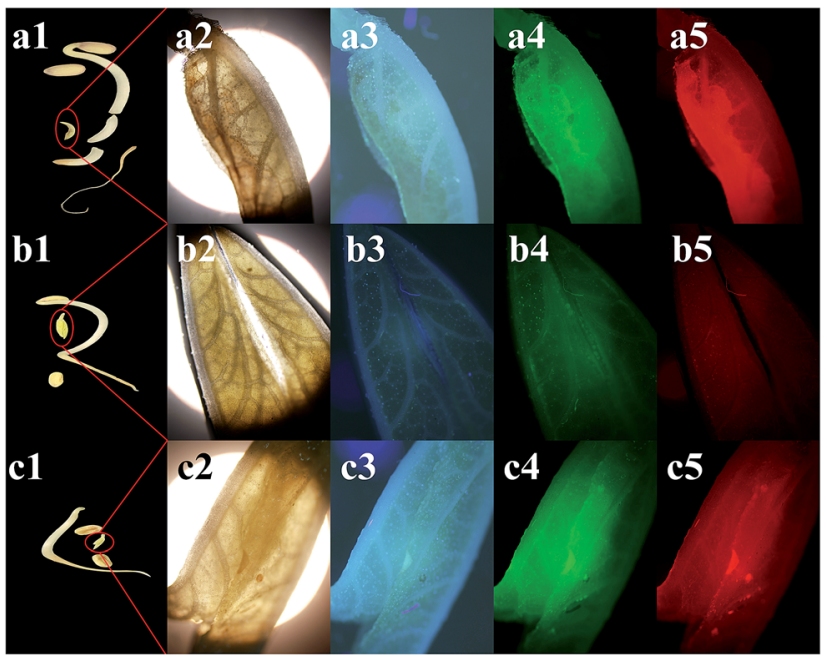

Fig. 8 Images of the mung bean sprouts under (a1, b1, and $c 1$ ) daylight, (a2, b2, and c2) bright field, (a3, b3, and c3) UV (405 nm), (a4, b4, and c4) blue (488 nm) and (a5, b5, and c5) green light (546 nm). (a, $b$ and $c$ ) Show the images of the mung bean sprouts incubated with the $\mathrm{N}-\mathrm{CD}$ solution, the $\mathrm{N}-\mathrm{CD}+\mathrm{Cr}(\mathrm{vI})$ solution and $\mathrm{N}-\mathrm{CD}+\mathrm{Cr}(\mathrm{vI})+\mathrm{AA}$ solution, respectively. The concentrations of the $\mathrm{N}-\mathrm{CDs}, \mathrm{Cr}(\mathrm{vI})$ and $\mathrm{AA}$ solution were $0.1 \mathrm{mg} \mathrm{mL}^{-1}, 50 \mu \mathrm{M}$, and $100 \mu \mathrm{M}$, respectively.

images of the mung bean sprouts with N-CDs as the fluorescent probe. The bean sprout produced cyan, green and red emission under the excitation of UV (405 $\mathrm{nm})$, blue $(488 \mathrm{~nm})$ and green (546 nm) light, respectively. When $\mathrm{Cr}(\mathrm{vI})$ entered the sprouts, the cyan, green and red emissions were significantly weakened (Fig. 8b3-b5). However, the emission could be recovered with the use of AA (Fig. 8c3-c5); this indicated that the N-CDs could be possibly employed as an efficient fluorescent probe for the "on-off-on" detection of $\mathrm{Cr}(\mathrm{vI})$ and AA in plants.

\section{Conclusion}

Herein, a novel solvent-free, solid-state and microwave-assisted approach was developed to synthesize fluorescent N-CDs with a high yield (58.5\%) using MA and urea as precursors. The reaction completed in three minutes, and no solvent was added. The N-CDs exhibited bright cyan emission with a high QY of $38.88 \%$ and showed excellent properties such as good solubility in water and excitation- and concentration-dependent properties. Based on the IFE, the as-prepared N-CDs could be employed as an "on-off" fluorescent sensor to detect $\mathrm{Cr}(\mathrm{vI})$ in water with a linear range of $1-100 \mu \mathrm{M}$ and an LOD of $0.12 \mu \mathrm{M}$, which was much lower than the US EPA stipulation $(1.9 \mu \mathrm{M})$. The quenched fluorescence has been successfully recovered ("off-on") with the addition of AA because AA has high reducibility towards $\mathrm{Cr}(\mathrm{vI})$; this results in the elimination of the IFE and recovery of the fluorescent N-CD system; furthermore, the photostability and low toxicity of the N-CDs successfully enable their application in bioimaging. Nowadays, the effect of $\operatorname{Cr}(\mathrm{vI})$ pollution is non-negligible, and the $\mathrm{Cr}(\mathrm{vI})$ detection methods are usually suffering from several restrictions: high cost, complicated operation, time-consuming detection, etc. CDs have predominant advantages in overcoming these limits. Of course, there are many CDs having lower LODs for the detection of $\mathrm{Cr}(\mathrm{vI})$; however, their preparation cost limits their further development. We believe that this study has made a comprehensive improvement in detection cost and accuracy. The proposed method could contribute to the research of CDs and fluorescent sensors for heavy-metal ion detection and bioimaging.

\section{Conflicts of interest}

The authors declare that they have no conflict of interest.

\section{Acknowledgements}

This research was supported by the National Key Research and Development Program of China (2016YFD0300801) and the National Natural Science Foundation of China (41571217).

\section{References}

1 S. Clemens and J. F. Ma, Annu. Rev. Plant Biol., 2016, 67, 489512.

2 G. Aragay, J. Pons and A. Merkoci, Chem. Rev., 2011, 111, 3433-3458.

3 E. Erdem, R. Böttcher, H.-J. Gläsel and E. Hartmann, Magn. Reson. Chem., 2005, 43, S174-S182.

4 E. Erdem, R. Böttcher, H. C. Semmelhack, H. J. Gläsel, E. Hartmann and D. Hirsch, J. Mater. Sci., 2003, 38, 32113217.

5 E. Erdem, R. Böttcher, H.-C. Semmelhack, H.-J. Gläsel and E. Hartmann, Phys. Status Solidi B, 2003, 239, R7-R9.

6 N. A. Qambrani, J.-H. Hwang and S.-E. Oh, Chemosphere, 2016, 160, 342-348. 
7 G. Hu, P. Li, X. Cui, Y. Li, J. Zhang, X. Zhai, S. Yu, S. Tang, Z. Zhao, J. Wang and G. Jia, Environ. Pollut., 2018, 238, 833-843.

8 D. Wang, G. Zhang, L. Zhou, M. Wang, D. Cai and Z. Wu, Langmuir, 2017, 33, 7007-7014.

9 United States Environmental Protection Agency (EPA), Chromium in Drinking Water, 2012, https:/www.epa.gov/ dwstandardsregulations/chromium-drinking-water.

10 S. M. Yousefi and F. Shemirani, J. Hazard. Mater., 2013, 254255, 134-140.

11 M. Hashemi and S. M. Daryanavard, Spectrochim. Acta, Part A, 2012, 92, 189-193.

12 M. Safari, S. Nojavan, S. S. H. Davarani and A. MortezaNajarian, Anal. Chim. Acta, 2013, 789, 58-64.

13 W. Jin, G. Wu and A. Chen, Analyst, 2014, 139, 235-241.

14 M. B. Gumpu, S. Sethuraman, U. M. Krishnan and J. B. B. Rayappan, Sens. Actuators, B, 2015, 213, 515-533.

15 X. Xu, R. Ray, Y. Gu, H. J. Ploehn, L. Gearheart, K. Raker and W. A. Scrivens, J. Am. Chem. Soc., 2004, 126, 12736-12737.

16 R. Genc, M. O. Alas, E. Harputlu, S. Repp, N. Kremer, M. Castellano, S. G. Colak, K. Ocakoglu and E. Erdem, Sci. Rep., 2017, 7, 11222.

17 X. T. Zheng, A. Ananthanarayanan, K. Q. Luo and P. Chen, Small, 2014, 11, 1620-1636.

18 S. Maiti, S. Kundu, C. N. Roy, T. K. Das and A. Saha, Langmuir, 2017, 33, 14634-14642.

19 G. Huang, X. Chen, C. Wang, H. Zheng, Z. Huang, D. Chen and H. Xie, RSC Adv., 2017, 7, 47840-47847.

20 X. Zhang, M. Jiang, N. Niu, Z. Chen, S. Li, S. Liu and J. Li, ChemSusChem, 2018, 11, 11-24.

21 S. Sun, K. Jiang, S. Qian, Y. Wang and H. Lin, Anal. Chem., 2017, 89, 5542-5548.

22 A. Simpson, R. R. Pandey, C. C. Chusuei, K. Ghosh, R. Patel and A. K. Wanekaya, Carbon, 2018, 127, 122-130.

23 S. Liu, J. Tian, L. Wang, Y. Zhang, X. Qin, Y. Luo, A. M. Asiri, A. O. Al-Youbi and X. Sun, Adv. Mater., 2012, 24, 2037-2041.

24 M. Zheng, Z. Xie, D. Qu, D. Li, P. Du, X. Jing and Z. Sun, ACS Appl. Mater. Interfaces, 2013, 5, 13242-13247.

25 D. K. Dang, C. Sundaram, Y.-L. T. Ngo, J. S. Chung, E. J. Kim and S. H. Hur, Sens. Actuators, B, 2018, 255, 3284-3291.

26 P. Wu, W. Li, Q. Wu, Y. Liu and S. Liu, RSC Adv., 2017, 7, 44144-44153.

27 H. Huang, Y. Weng, L. Zheng, B. Yao, W. Weng and X. Lin, J. Colloid Interface Sci., 2017, 506, 373-378.

28 Q. Wu, X. Wang, S. A. Rasaki, T. Thomas, C. Wang, C. Zhang and M. Yang, J. Mater. Chem. C, 2018, 6, 4508-4515.

29 H. Yang, L. He, Y. Long, H. Li, S. Pan, H. Liu and X. Hu, Spectrochim. Acta, Part A, 2018, 205, 12-20.

30 S. Qu, X. Wang, Q. Lu, X. Liu and L. Wang, Angew. Chem., 2012, 51, 12215-12218.

31 S. Qu, D. Shen, X. Liu, P. Jing, L. Zhang, W. Ji, H. Zhao, X. Fan and H. Zhang, Part. Part. Syst. Charact., 2014, 31, 1175-1182.
32 C. Zhang, Y. Cui, L. Song, X. Liu and Z. Hu, Talanta, 2016, 150, 54-60.

33 E. Bezdushna and H. Ritter, Macromol. Rapid Commun., 2005, 26, 1087-1092.

34 J. Lu, X. Zhu, S. Ji, J. Zhu and Z. Chen, J. Appl. Polym. Sci., 1998, 68, 1563-1566.

35 Z. Wang, F. Yuan, X. Li, Y. Li, H. Zhong, L. Fan and S. Yang, Adv. Mater., 2017, 1702910.

36 H. Zhang, Y. Huang, Z. Hu, C. Tong, Z. Zhang and S. Hu, Microchim. Acta, 2017, 184, 1547-1553.

37 F. Akhgari, N. Samadi and K. Farhadi, J. Fluoresc., 2017, 27, 921-927.

38 C. Tan, X. Su, C. Zhou, B. Wang, Q. Zhan and S. He, RSC Adv., 2017, 7, 40952-40956.

39 J. Yu, N. Song, Y.-K. Zhang, S.-X. Zhong, A.-J. Wang and J. Chen, Sens. Actuators, B, 2015, 214, 29-35.

40 S. Lu, L. Sui, J. Liu, S. Zhu, A. Chen, M. Jin and B. Yang, Adv. Mater., 2017, 29, 1603443.

41 W. Lu, X. Qin, S. Liu, G. Chang, Y. Zhang, Y. Luo, A. M. Asiri, A. O. Al-Youbi and X. Sun, Anal. Chem., 2012, 84, 5351-5357.

42 X. Zhang, H. Wang, C. Ma, N. Niu, Z. Chen, S. Liu, J. Li and S. Li, Mater. Chem. Front., 2018, 2, 1269-1275.

43 Y. Wang, S. Kalytchuk, Y. Zhang, H. Shi, S. V. Kershaw and A. L. Rogach, J. Phys. Chem. Lett., 2014, 5, 1412-1420.

44 Y. Chen, H. Lian, Y. Wei, X. He, Y. Chen, B. Wang, Q. Zeng and J. Lin, Nanoscale, 2018, 10, 6734-6743.

45 Y. Chen, M. Zheng, Y. Xiao, H. Dong, H. Zhang, J. Zhuang, H. Hu, B. Lei and Y. Liu, Adv. Mater., 2016, 28, 312-318.

46 J. Wu, W. Liu, J. Ge, H. Zhang and P. Wang, Chem. Soc. Rev., 2011, 40, 3483-3495.

47 M. Lan, Y. Di, X. Zhu, T.-W. Ng, J. Xia, W. Liu, X. Meng, P. Wang, C.-S. Lee and W. Zhang, Chem. Commun., 2015, 51, 15574-15577.

48 X. Wu, Y. Song, X. Yan, C. Zhu, Y. Ma, D. Du and Y. Lin, Biosens. Bioelectron., 2017, 94, 292-297.

49 S. Chen, Y.-L. Yu and J.-H. Wang, Anal. Chim. Acta, 2018, 999, 13-26.

50 X.-R. Xu, H.-B. Li, J.-D. Gu and X.-Y. Li, Environ. Toxicol. Chem., 2005, 24, 1310-1314.

51 H.-Y. Zhang, Y. Wang, S. Xiao, H. Wang, J.-H. Wang and L. Feng, Biosens. Bioelectron., 2017, 87, 46-52.

52 J. Chen, J. Liu, J. Li, L. Xu and Y. Qiao, J. Colloid Interface Sci., 2017, 485, 167-174.

53 H.-Y. Li, D. Li, Y. Guo, Y. Yang, W. Wei and B. Xie, Sens. Actuators, B, 2018, 277, 30-38.

54 J. Shen, S. Shang, X. Chen, D. Wang and Y. Cai, Sens. Actuators, B, 2017, 248, 92-100.

55 X. Liu, T. Li, Q. Wu, X. Yan, C. Wu, X. Chen and G. Zhang, Talanta, 2017, 165, 216-222.

56 J. Wang and J. Qiu, J. Mater. Sci., 2016, 51, 4728-4738. 